\title{
Constraining the network topology in IEEE 802.15.4
}

\author{
Anna Abbagnale, Emanuele Cipollone, Francesca Cuomo
}

\begin{abstract}
The IEEE 802.15.4 standard defines a MAC association procedure that allows devices of a Wireless Personal Area Network (WPAN) to associate one each other in accordance to parent-child relationships. This standard can be adopted in Wireless Sensor Networks (WSNs) used to monitor environmental phenomena and to collect data in specific nodes named sinks. The ZigBee Alliance, with the ZigBee Specification, defines upper layers of a WSN architecture based on the IEEE 802.15.4. Tree shaped multi-sink networks can be formed by adopting in conjunction these two standards. A key aspect to be evaluated for these kind of networks is how their performance are affected by constraining some topological characteristics. In this paper, in accordance to the ZigBee Specification, we constraint the tree depth of a WSN and evaluate some performance metrics that have a remarkable relevance for WSNs. Moreover, we propose a new strategy to join an existing WPAN that allows a node to select a suitable coordinator to connect with. Performance results show the trade-off in the selection of the maximum tree depth. While benefits of having reduced tree depths can be measured during traffic delivery, due to a reduction of the energy consumption, a counter-party is the energy spent in the network formation that deeply depends on this topological parameter. Moreover, the maximum tree depth has also a great impact on the percentage of nodes that are able to join a network.
\end{abstract}

Key words: IEEE 802.15.4, ZigBee, Sensor Networks, Topology Formation.

\section{Introduction}

Wireless Sensor Networks (WSNs) are traditionally composed of a multiplicity of sensor nodes that sense physical or environmental phenomenona (e.g. atmospheric

Anna Abbagnale, Emanuele Cipollone, Francesca Cuomo

University of Rome "Sapienza", INFOCOM Dpt, via Eudossiana 18, 00184 Rome (Italy)

Please use the following format when citing this chapter:

Abbagnalc, A., Cipollone, E., Cuomo, F., 2008, in IFIP International Federation for Information Processing, Volume 265, Advances in Ad Hoc Networking, eds. Cuenca, P., Guerrero C., Puigjaner, R., Serra, B., (Boston: Springer), pp. $167-178$ 
pressure, temperature, sound, vibration, etc.) and generate samples of them, that have to be delivered, possibly through multi-hop paths, to a specific node (named sink) collecting the information $[1,2]$. Generally, sensor nodes are battery powered, whereas sink is connected to an outlet.

The IEEE 802.15.4 standard [3] can be used as basic technology for the development of low energy and low cost WSNs. It defines physical and MAC (Medium Access Control) layers, while the upper layers of the protocol stack are specified by the ZigBee Alliance [4]. Recently, some papers have treated these standards by presenting potentialities [5] and performance [6,7]. The paper of Baronti et al. [6] presents an overview of the energy efficiency, communication, data management and security solutions adopted by the standards and proposed in the recent literature.

The paper in [7] discusses results derived by simulating, in $n s-2$ [8], the IEEE 802.15.4 to (1) compare the performance between 802.15 .4 and 802.11 ; (2) study the association and tree formation; (3) investigate the orphaning and coordinator relocation; (4) exam unslotted CSMA-CA and slotted CSMA-CA behaviors; and (5) compare three different data transmissions, namely, direct, indirect and with guaranteed time slot (GTS).

Another key aspect that should be analyzed is how this technology behaves when the applications require the set-up of a network topology with attention to the energy efficiency and network connectivity. To set-up the network topology, the IEEE 802.15.4 defines a formation strategy (MAC association procedure) that forms WPANs coordinated by a node (the sink) named PAN coordinator. Nodes associated to a single PAN coordinator are arranged in a tree in accordance to parent-child relationships. In an IEEE 802.15.4 WSN, it is possible to have Full Function Devices (FFDs), that allow the association of other nodes to the network and Reduced Function Devices (RFDs), that do not permit the association of other nodes. The sink is always a FFD, intermediate nodes allowing data relay (router) are FFDs too, whereas the RFDs are the leaves of the tree. The level of a node in the tree is intended as the distance (in terms of number of hops) of the node from the sink. Node belonging to level $d$ are named $d$-nodes, so nodes at level $d=0$ are sinks, nodes at level $d=1$ are directly connected to the sinks, nodes at level $d=2$ are at a two-hop distance from the sink, etc.

In the same region it is possible to have more than one sink. In this case each sink is a PAN coordinator and the overall network topology results in a forest of disjoint trees. This is a multi-sink network. This type of network overcomes the following problems of single-sink (or single-tree) architectures:

- when the number of sensor nodes increases, the sink has to collect an amount of information that may exceed its data processing capability (scalability problem);

- if in a tree several nodes belong to high levels, the delivery of the samples to the sink involves a large number of nodes, so the network energy drastically reduces (energy consumption problem).

In the paper in [9] the topologies formed by means of the IEEE 802.15.4 MAC association procedure are investigated. Specific attention is dedicated to the characterization of network lifetime and event notification reliability for different sinks 
distribution and varying the range of the event detection. Multi-sink topology performance is compared with classical single sink ones. This topological analysis highlighted that some topologies are not energy efficient, since very high trees are formed; these results could be used to better design the IEEE 802.15.4 association procedure.

Besides the possibility to have multi-sinks, it is interesting to evaluate also the impact on the performance of the tree characteristics. In particular, a key parameter to be controlled is the tree depth defined as the maximum level that a node can have in the tree. The use of this parameter can affect the network performance, since it shapes the structure of the topologies resulting from the IEEE 802.15.4 association procedure. In this paper we aim at considering both the multi-sink capability and the constraint on the tree depth to evaluate the network topology performance. The work of [10] aims at proposing a model to optimize the network formation by constraining the tree depth. Here we perform a detailed analysis on constrained (in terms of depth) network topologies in order to support the network optimization.

The paper is organized as follows: Section 2 briefly describes the motivations of our work. Section 3 focuses on the association procedure defined in the IEEE 802.15.4 to form a connected network. In Section 4 we show and discuss the results of the simulation analysis. Finally, the overall conclusions of this analysis are provided in Section 5.

\section{Motivations of our work}

The possibility to have trees with controlled topological characteristics has been addressed by the ZigBee Alliance. In the ZigBee Specifications the following parameters are defined [4]:

- nwkMaxDepth (Lm), the maximum depth in the network, that is the maximum number of levels of the tree;

- nwkMaxChildren $(\mathrm{Cm})$, the maximum number of children a parent may have;

- nwkMaxRouters $(\mathrm{Rm})$, the maximum number of children with the role of router a parent may have.

These parameters are determined by the ZigBee coordinator which corresponds to an IEEE 802.15.4 PAN coordinator. Using these parameters every node, able to be parent, calculates the addresses to be assigned to its children. The size of the addresses block depends on the level of the node in the tree.

The aim of this work is to evaluate pros and cons that the network formation may have when the parameter $\mathrm{Lm}$ is taken into account. This analysis can be used as a basis for works that analyze the performance of the ZigBee networks (e.g. the paper [11]), where the tree creation is achieved by implementing the ZigBee recommendations as for the tree parameters. Some recent works analyzed also the ZigBee network dimensioning as a function of the tree parameters [12]. Also in this case a deep analysis of the tree topologies characteristics is fundamental to dimension the 
network resources. In this paper we propose a new strategy that allows a node to join an existing WPAN and takes into account the constraint on the $L m$ parameter. We then analyze the impact of this parameter on the following metrics:

- percentage of nodes connected to the network;

- percentage of energy consumption for network formation;

- percentage of energy consumption for packet delivery.

The first metric has an impact on the coverage of the reference area and on the reliability of event detection. The other ones are important because energy saving is a fundamental goal for WSNs. It is important to notice that the energy consumption for network formation is often not considered in literature and the networks are analyzed from the time instant in which they are already formed.

\section{IEEE 802.15.4 Topology Formation}

An IEEE 802.15.4 WPAN [3] is composed of one PAN coordinator and a set of sensor nodes. The PAN coordinator is the primary controller of the network and it is responsible for initiating the network operations. The standard defines a set of procedures implemented by the PAN coordinator to initiate a new WPAN and by other nodes to join an existing WPAN. The PAN coordinator assigns a PAN ID (PAN identifier) to the network and selects a channel among those specified in the standard.

The procedure adopted by sensor nodes to join a WPAN is named association procedure and it establishes relationships between devices within a WPAN. The operations performed by a node to join a WPAN are: STEP 1) the node searches for the available WPANs, STEP 2) it selects a coordinator ${ }^{1}$ belonging to the available WPANs and STEP 3) it starts a message exchange with the selected coordinator to associate with it.

The discovery of available WPANs (STEP 1) is performed by scanning the beacon frames broadcasted by the coordinators. Two beacon broadcasting modes are defined in the standard: beacon-enabled and nonbeacon-enabled.

In beacon-enabled mode, the associated nodes transmit beacon frames periodically, hence the information on the available WPANs can be derived by eavesdropping the wireless channels (passive scan). In nonbeacon-enabled mode, the beacon frames shall be explicitly requested by a node by means of a beacon request command frame (active scan).

After the channels scan, the sensor node selects the coordinator (and, therefore, implicity the WPAN) to connect to (STEP 2) and it sends an association request message to the chosen coordinator (STEP 3). The coordinator grants and denies the access to the network of the new node by replying with an association response command frame. It is important to notice that the criterion used to select the coordinator (STEP 2) is not defined in the standard but is implementor dependent.

\footnotetext{
${ }^{1}$ Coordinators are sinks or those nodes that can act as relay nodes.
} 
The whole association procedure results in a set of parent-child relationships between nodes. These relationships define univocally a tree rooted at the PAN coordinator.

\subsection{Proposed strategy for coordinator selection}

In this subsection' we describe our implementation of the coordinator selection (STEP 2 of the association procedure) suitably designed to take into account the multi-sink feature and the constraint on the maximum number of levels of the trees (Lm parameter).

In Algorithm 1, we report the pseudo-code of the operations which are executed by a node trying to connect to a network. A generic node in the network is indicated as $x$. Nodes that are coordinators are indicated as $C$ and the level within the tree where they belong is indicated as $d_{C}$. A generic node $x$ begins the association procedure with the channels scan procedure (STEP 1) as described before and it is able to listen a number of coordinators equal to numCoord; so, a possible coordinator for the node $x$ is indicated as $C^{(j)}$, with $j=1, \ldots$, numCoord and its level is $d_{C^{(j)}}$. With $L Q I_{C^{(j)}}$ we indicate, in accordance with the IEEE 802.15.4 standard, the quality of the link between the coordinator $C^{(j)}$ and the node $x$.

Among the coordinators found by node $x$ (lines $4-5$ ), the selection of the coordinator (STEP 2) is performed by means of the following operations:

- node chooses the coordinator having the lowest level $d_{C^{(j)}}$ (lines from 14 to 17);

- if there are two or more coordinators belonging to the lowest level, among the numCoord coordinators, the node chooses the one with the highest value of the $L Q I$ parameter (lines from 23 to 26);

- if there are two or more coordinators belonging to the lowest level and having the same value of the $L Q I$ parameter, the node chooses randomly one of them (lines from 31 to 36 ).

In all the three cases, the chosen coordinator is really selected if it has a level less than the $n w k M a x D e p t h(L m)$ parameter.

\section{Performance analysis}

\subsection{Simulation model}

We consider a multi-sink scenario with $N$ sensor nodes and $S$ sinks, randomly deployed in a square of side $L$ and area $A=L^{2}$. All nodes are motionless and FFDs (this means that they act as possible coordinators in each WPAN, allowing association to other nodes). The transmission range of each device is $R$. We hypothesize that the considered scenario is static, that is node/link failures cannot occur. However at 
MAC level we assume that beacon frames and data packets can collide, since they are transmitted in accordance with the CSMA-CA protocol as defined in the IEEE 802.15.4 standard. The $N$ nodes operate in nonbeacon-enabled mode, while the $S$ sinks are configured in beacon-enabled mode. As for the propagation model, the two-ray ground reflection one [13] is used; it contemplates both the direct path and one ground reflection path. We suppose that sinks have infinite energy, because they are connected to an outlet, whereas the initial energy of the other nodes is set to $1 J$. We assume that the energy spent during the transmission is equal to the one spent for the reception and this value is set to $0,39 \mu \mathrm{J} /$ bit [15]. The adopted routing protocol is HERA (HiErarchical Routing Algorithm) [14]: this is a hierarchical protocol, where data generated by sensors and directed to the sink are routed along the parent-child relationships, i.e., every node relays data to its parent.

We performed simulations using a modified version of the $n s-2$ module originally provided by Zheng and Lee in [8]. In particular, we extended the original version of this simulator (that implements the association procedure provided by the IEEE 802.15.4 Standard), to simulate IEEE 802.15.4 multi-sink networks with a constraint on the maximum number of levels of the trees, represented by the $n w k$ MaxDepth $(\mathrm{Lm})$ parameter. At the beginning of the simulation nodes try to join a network, therefore during this phase the percentage of nodes connected to a network increases up to reach a specific value. When this value steadies, we stop the association procedure for all nodes. From this time instant (named in the following $t_{I N}$ ) nodes connected to the network start to generate traffic. During each IEEE 802.15.4 superframe each sensor node transmits one packet (19 bytes at MAC level): the used traffic model is the Constant Bit Rate (CBR) one.

In Table 1 all the simulation parameters are summarized.

Table 1 Simulation assumptions and parameters

\begin{tabular}{|c|c|c|c|}
\hline Parameter & Value & Parameter & Value \\
\hline \hline Number of sinks, $S$ & $25,30,35,40,45,50$ & Initial energy of the sensor nodes & $1 \mathrm{~J}$ \\
Number of sensor nodes, $N$ & 1500 & Transmission energy $E_{T X}$ & $0,39 \mu \mathrm{J} / \mathrm{bit}$ \\
Side of the square area, $L$ & $1000 \mathrm{~m}$ & Reception energy $E_{R X}$ & $0,39 \mu \mathrm{J} / \mathrm{bit}$ \\
Radio transmission range, $R$ & $150 \mathrm{~m}$ & Routing protocol & HERA [14] \\
Propagation model & Two-ray ground & Traffic model & CBR \\
\hline
\end{tabular}

\subsection{Simulation metrics}

In this Subsection we formally define the three metrics introduced in the Section 2.

The percentage of nodes connected to the network is the ratio between the number of sensor nodes connected to the network $\left(N_{c}\right)$ at the end of the association procedure (that is at the time instant $t_{I N}$ of Fig. 1) and the total number of sensor nodes $(N)$. So, this metric is calculated as: 


$$
\text { Percentage of nodes connected to the network }(\%)=\frac{N_{c}}{N}
$$

The percentage of energy consumption for network formation is the percentage of energy that a node spends on average to join the network, compared with its initial energy. We indicate with $x^{(k)}$ a generic node, where $k=0,1, \ldots, N-1$. If $E_{x^{(k)}}^{I}$ is the initial energy of $x^{(k)}$ and $E_{x^{(k)}}^{T}$ is the residual energy of the same node at the time instant $t_{I N}$, the percentage of spent energy by $x^{(k)}\left(E_{x^{(k)}}^{N F}\right)$ is calculated as:

$$
E_{x^{(k)}}^{N F}(\%)=\frac{E_{x^{(k)}}^{I}-E_{x^{(k)}}^{T}}{E_{x^{(k)}}^{I}}, \quad k=0, \ldots, N-1
$$

The result of the equation 2 is averaged on all the $N$ sensor nodes to obtain the percentage of energy consumption for network formation, $E_{N F}$.

The percentage of energy consumption for packet delivery is the percentage of energy that a node spends on average, during a superframe, to support a CBR traffic. During a supertrame a generic node consumes energy to transmit its packet and to receive and relay packets transmitted by other nodes.

In Fig. 1 is represented the time axis, where:

- $t_{I N}$ is the time instant in which we stop the association procedure and sensor nodes connected to the network start to transmit periodically their packets;

- $E_{x^{(k)}}^{S}$ is the energy that a generic node $x^{(k)}$ (with $k=0,1, \ldots, N_{c}-1$ ) has in the time instant $t_{I N}+\delta_{I N}$ (where the first superframe starts after $t_{I N}$ );

- $t_{F I N}$ is the time instant in which the network is declared dead (this happens when the first node exhausts its energy);

- $E_{x^{(k)}}^{F}$ is the energy that a generic node $x^{(k)}$ (with $k=0,1, \ldots, N_{C}-1$ ) has in the time instant $t_{F I N}-\delta_{F I N}$ (where the last superframe ends before $t_{F I N}$ ).

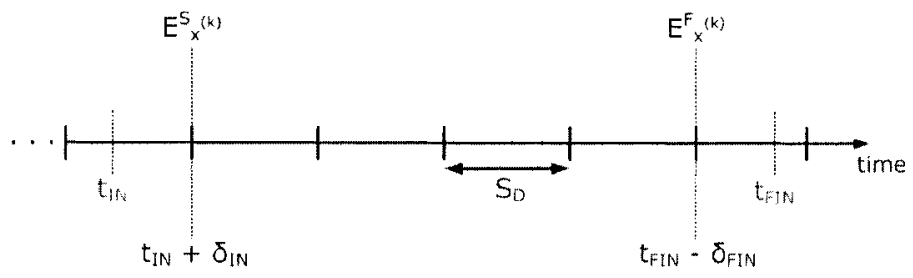

Fig. 1 Time axis partition in superframes.

If $S_{D}$ is the superframe duration, the number of superframe $M$ contained in the time interval $\left[t_{I N}+\delta_{I N}, t_{F I N}-\delta_{F I N}\right]$ is calculated as:

$$
M=\frac{\left(t_{F I N}-\delta_{F I N}\right)-\left(t_{I N}+\delta_{I N}\right)}{S_{D}}
$$


The energy spent by the node $x^{(k)}$ during a superframe $\left(E_{x^{(k)}}^{S_{D}}\right)$ is, therefore, calculated as:

$$
E_{x^{(k)}}^{S_{D}}=\frac{E_{x^{(k)}}^{S}-E_{x^{(k)}}^{F}}{M}, \quad k=0, \ldots, N_{c}-1
$$

To obtain the correspondent value in percentage, we divide the result of the equation 4 for the initial energy of the node $x^{(k)}$ :

$$
E_{x^{(k)}}^{S_{D}}(\%)=\frac{E_{x^{(k)}}^{S_{D}}}{E_{x^{(k)}}^{I}}, \quad k=0, \ldots, N_{c}-1
$$

The result of the equation 5 is averaged on all the $N_{c}$ sensor nodes, to obtain the percentage of energy consumption for packet delivery, $E_{P D}$.

\subsection{Simulation results}

In Fig. 2 it is shown the percentage of nodes connected to the network as function of $S$ for different values of $L m$. It is important to notice that this percentage nodes increases with $L m$. For low values of $L m$, a reduced number of nodes succeeds in the association. This phenomenon is attenuated if $S$ increases. However, when the value of $L m$ is low (e.g. $L m=3$ ) the percentage of nodes connected to the network remains low even if $S$ is high. This is due to the fact that in this case some nodes perform several attempts to connect to the network, thus causing several MAC collisions and, hence, exhausting completely their energy before successfully joining the network. The difference in the percentage of nodes connected to the network for values of $L m \geq 9$ is low. In general, when $S$ increases, the percentage of connected nodes is high, since the nodes deployed in the reference area can distribute on a greater number of trees.

In Fig. 3 it is shown $E_{N F}$ as function of $S$ for different values of $L m$. When the value of $L m$ is low, $E_{N F}$ is high. This effect is due to two main contributions:

- the maximum depth of the trees is reached with a minor number of connected nodes, therefore there are several nodes that repeatedly try to join the network without success (contribution in the spent energy of nodes not associated to the network);

- the number of attempts performed by a node to select a suitable coordinator (and therefore to join successfully the network) increases, due to constraint on $\mathrm{Lm}$ (contribution in the spent energy of nodes associated to the network).

On the other hand, we can notice that when $S$ varies, this performance metric remains nearly constant. This result could not seem intuitive, because we expect a decrease of $E_{N F}$ when $S$ increases. However, it is to be noticed that when $S$ increases, also the number of transmitted beacons increases; as a consequence, MAC collisions and the energy spent to transmit and to receive beacons rise too. 


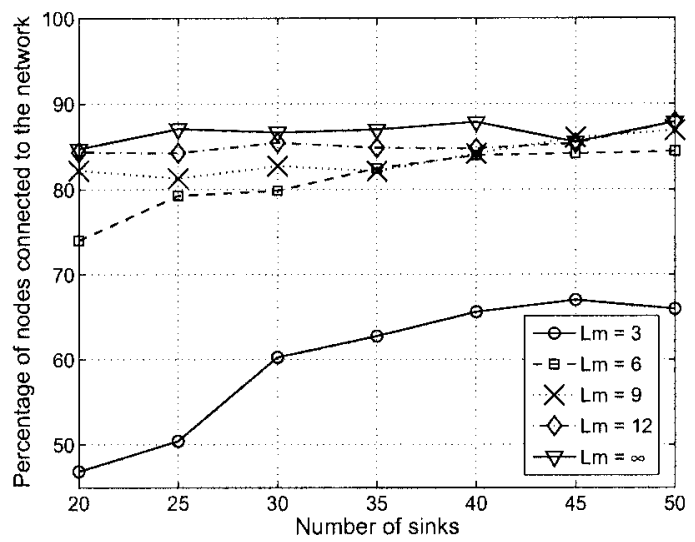

Fig. 2 Percentage of nodes connected to the network as function of number of sinks for different values of the $L m$ parameter.

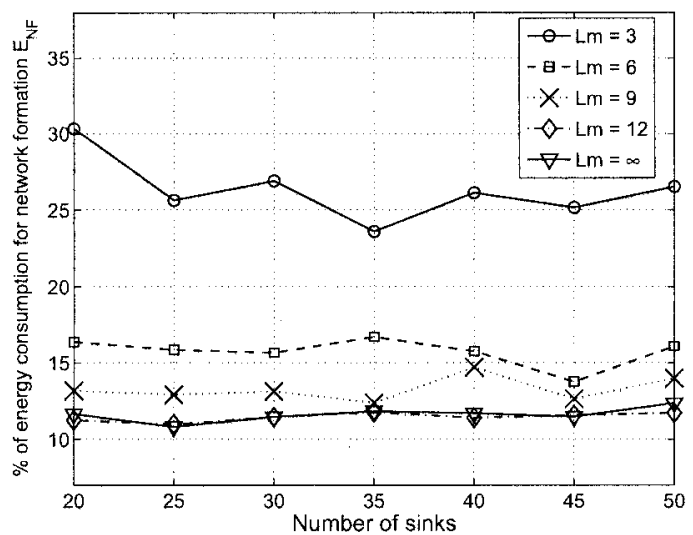

Fig. 3 Percentage of spent energy for network formation as function of number of sinks for different values of the $L m$ parameter.

In Fig. 4 it is shown $E_{P D}$ as function of $S$ for different values of $L m$. In this case there is an opposite trend with respect to the Fig. 3. In fact when $\mathrm{Lm}$ is low, $E_{P D}$ decreases, because the number of hops to reach the sink decreases too (in accordance to the used routing protocol). Moreover, if the value of $L m$ is high (e.g., $L m \geq 6$ ), $E_{P D}$ decreases when $S$ increases. The reason is that the nodes can distribute on a greater number of trees and so some of them could have a maximum depth less than Lm, guaranteeing that data packets reach the sinks with a minor number of hops. 


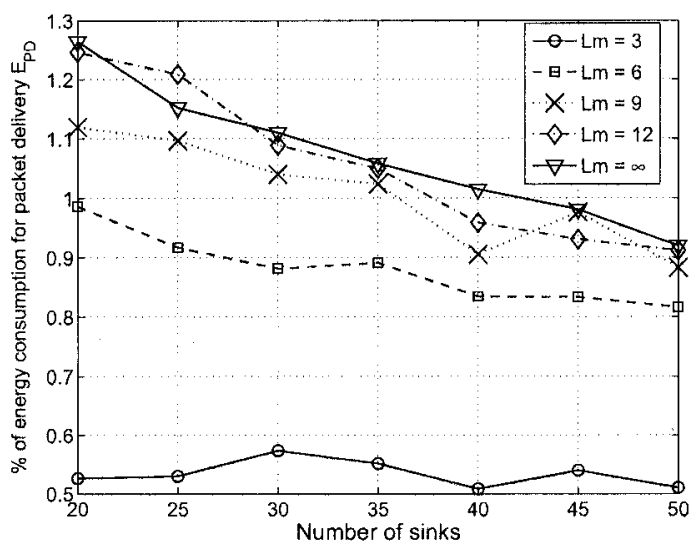

Fig. 4 Percentage of spent energy for packet delivery as function of number of sinks for different values of the $L m$ parameter.

\section{Overall Results and Conclusions}

In this paper, we analyzed tree shaped multi-sink networks, which form by adopting in conjunction the IEEE 802.15.4 standard and the ZigBee Specification. We evaluated how the performance of this kind of networks is affected, in terms of connectivity and energy consumption, by imposing a constraint on the maximum depth of the network $(\mathrm{Lm})$.

Performance results show the trade-off that can arise in the selection of this parameter. While benefits of having reduced tree depths can be measured during traffic delivery, due to a reduction of the energy consumption, a counter-party is the energy spent in the network formation that deeply depends on this topological parameter. Moreover, the maximum tree depth has also a great impact on the percentage of nodes that are able to connect in a network. A very low number of connected nodes is achieved if the $\mathrm{Lm}$ parameter is maintained low. This effect can only partially be alleviated by increasing the number of sinks in the network.

The achieved results suggest that it is very important to choose a suitable value of $\mathrm{Lm}$. It depends on characteristics of the WSN architecture (e.g., in terms of number of sinks) and on application requirements. For applications that need low routing delays and/or low energy consumption for packet delivery, it might be thought to choose low values of $L m$, but only if this choice does not imply a significant loss of coverage. On the other hand for applications that need a high reliability in events notification to sink it seems more convenient to select an high $\mathrm{Lm}$, since this allows to obtain high values of connectivity and a good coverage of the area to be monitored. Therefore a suitable value of this parameter has to be chosen in order to achieve the best trade-off among network performance of interest. 


\section{Acknowledgment}

This work has been partially supported by the EC-funded Network of Excellence CRUISE, in the framework of the FP6.

A special thanks goes to Fabio Trifiró for the support in the simulations.

\section{References}

1. H. Karl, A. Willig, "Protocols and Architectures for Wireless Sensor Networks", Wiley, 2005.

2. I. F. Akyildiz, W. Su, Y. Sankarasubramaniam, E. Cayirci, "A Survey on Sensor Networks", IEEE Communications Magazine, Aug. 2002, pp. 102-114.

3. IEEE standard: Part 15.4: Wireless Medium Access Control (MAC) and Physical Layer (PHY) Specifications for Low-Rate Wireless Personal Area Networks (WPANs), IEEE, 2006.

4. ZigBee Specification, 2006, Zigbee Alliance, available at http://www.zigbee.org.

5. J. Gutierrez, E. Callaway, R. Barret, "Low-Rate Wireless Personal Area Networks - Enabling Wireless Sensors with IEEE 802.15.4", IEEE Press, 2003.

6. P. Baronti, P. Pillaia, V.W.C. Chooka, S. Chessa, A. Gottab, Y. Fun Hua, "Wireless sensor networks: A survey on the state of the art and the 802.15.4 and ZigBee standards", Elsevier Computer Communications 30, 2007, pp. 1655-1695.

7. J. Zheng, M. Lee, "A Comprehensive Performance Study of IEEE 802.15.4", IEEE press 2004.

8. J. Zheng, M. Lee, "NS2 Simulator for IEEE 802.15.4", http://ees2cy.engr.ccny.cuny.edu/zheng/pub/, 2004.

9. E. Cipollone, F. Cuomo, S. Della Luna, U. Monaco, F. Vacirca, "Topology Characterization and Performance Analysis of IEEE 802.15.4 Multi-Sink Wireless Sensor Networks", MedHocNet 2007, Corfu' (Greece), June 13-15 2007.

10. C. Buratti, F. Cuomo, S. Della Luna, U. Monaco, J. Orriss, R. Verdone, "Optimum TreeBased Topologies for Multi-Sink Wireless Sensor Networks Using IEEE 802.15.4", IEEE VTC 2007-Spring, pp. 22-25, Dublin (Ireland), April 2007.

11. F. Claudios, R. Radeke, D. Marandin, "Performance Study of Reconfiguration Algorithms in Cluster-Tree Topologies for Wireless Sensor Networks", PIMRC 2007, September 2007.

12. A. Koubaa, M. Alves, E. Tovar, "Modeling and Worst-Case Dimensioning of Cluster-Tree Wireless Sensor Networks", RTSS'06, pp. 412-421, 2006.

13. "The Network Simulator manual", http://www.isi.edu/nsnam/ns/ns-documentation, 2005.

14. F. Cuomo, S. Della Luna, U. Monaco, T. Melodia, "Routing in ZigBee: Benefits from Exploiting the IEEE 802.15.4 Association Tree", ICC 2007, June 2007.

15. Moteiv Corporation Website, http://www.moteiv.com/community/Tmote_Sky_Downloads 


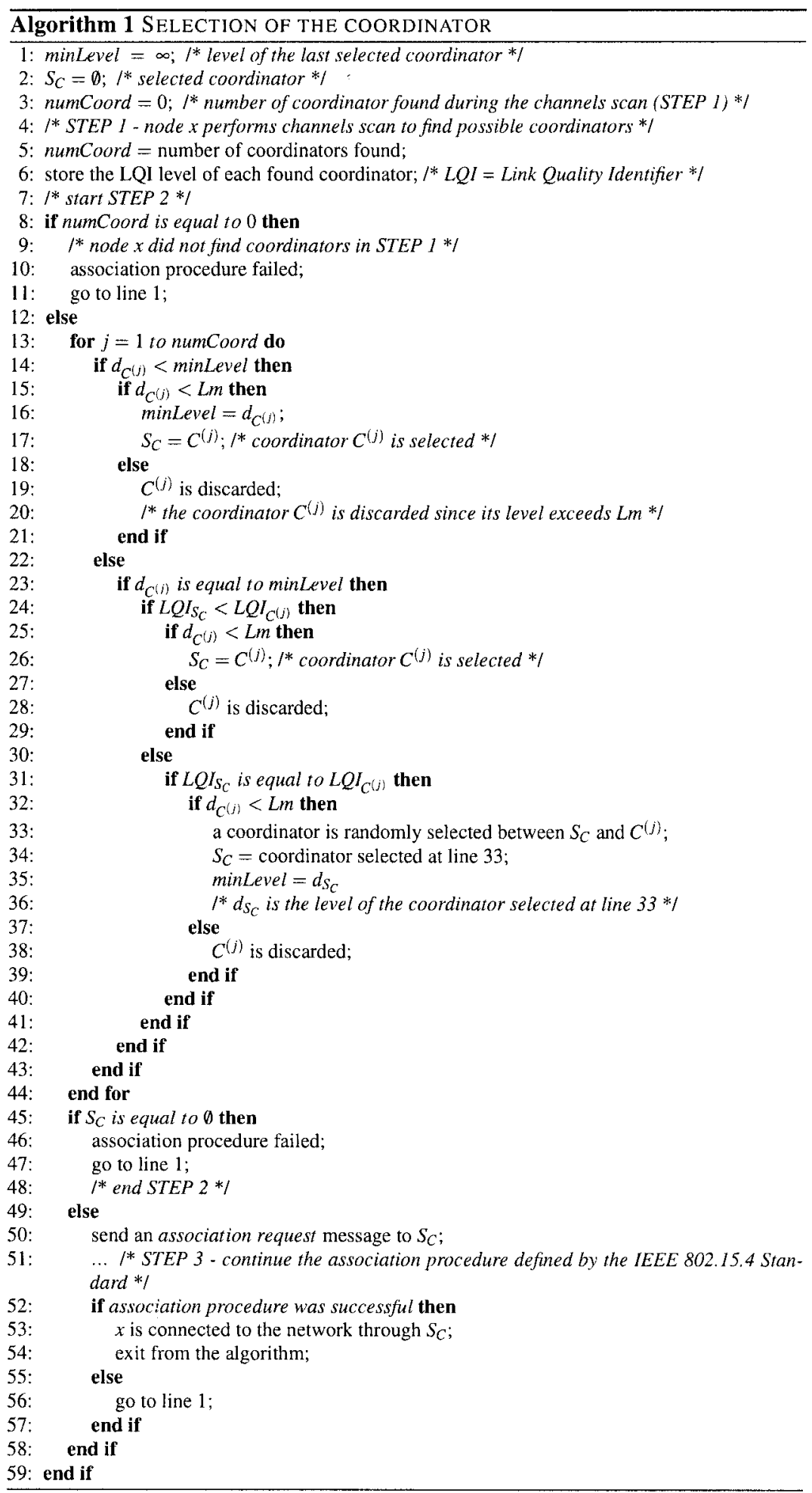

